\title{
A CLASS OF THE QUASILINEAR PARABOLIC SYSTEMS ARISING IN POPULATION DYNAMICS *
}

\author{
YANG WANLI ${ }^{\dagger}$
}

\begin{abstract}
This paper,using the duality technique and Hölder's inequality, proves the global existence of solutions for the quasilinear parabolic systems with Cross-diffusion effects and compectition interaction on any smooth bounded domain in $R^{2}$.
\end{abstract}

1. Introduction. The purpose of the present paper is to study the existence and uniqueness of $W_{p}^{2,1}$ valued solutions for quasilinear parabolic systems arising in population dynamics as

$$
\begin{cases}u_{t}=\triangle\left(a_{1} u+d u v\right)+u F(u, v), & (x, t) \in \Omega \times(0, \infty) . \\ v_{t}=\triangle\left(a_{2} v\right)+v G(u, v), & (x, t) \in \Omega \times(0, \infty) . \\ \delta u+(1-\delta) u_{\gamma}=0, & (x, t) \in \partial \Omega \times(0, \infty) . \\ \delta v+(1-\delta) v_{\gamma}=0, & (x, t) \in \partial \Omega \times(0, \infty) . \\ u(x, 0)=u_{0}(x), \quad v(x, 0)=v_{0}(x), & x \in \Omega .\end{cases}
$$

Here, $\Omega$ is a bounded domain in $R^{2}$ with smooth boundary $\partial \Omega ; a_{i}(i=1,2), d$ are positive constants; $F, G$ are given $\mathrm{C}^{1}$-functions; $\delta \in\{0,1\} ; \gamma$ denoting the outer unit normal on $\partial \Omega ; u_{0}$ and $v_{0}$ are initial functions which are assumed to satisfy

$$
\left\{\begin{array}{ll}
u_{0}, v_{0} \in H^{1+\varepsilon}(\Omega) \quad \text { for some } & 0<\varepsilon<1 ; \\
u_{0}(x), v_{0}(x) \geq 0, & \forall x \in \Omega ;
\end{array} \quad\left(H_{0}\right) .\right.
$$

This systems has been introduced by Shigesada et al.[1] as a model of two competitive species which are interacting each other and migrating under self and crossdiffusion effects. The unknown functions $u$ and $v$ denote the population densities of the two species at time $t$ and position $x \in \Omega$. The two boundary conditions show that the flow of an individual is tangential on the boundary $\partial \Omega$.

Masuda and Mimura (cf. [2]) proved, for the first time, global existence of a solution in the case that $\Phi \equiv 0$ and $\Omega \subset R^{1}$.

Yagi [3] studied the following systems.

$$
\left\{\begin{array}{rlrl}
u_{t}= & \operatorname{div}\left\{\nabla\left[\left(\alpha_{1}+\alpha_{11} u+\alpha_{12} v\right) u\right]\right. & \\
& \left.+\beta_{1} u \nabla \Phi\right\}+c_{1} u-\gamma_{11} u^{2}-\gamma_{12} u v, & & \text { in } \Omega \times(0, \infty), \\
v_{t}= & \operatorname{div}\left\{\nabla\left[\left(\alpha_{2}+\alpha_{21} u+\alpha_{22} v\right) v\right]\right. & & \\
& \left.+\beta_{2} v \nabla \Phi\right\}+c_{2} v-\gamma_{21} u v-\gamma_{22} v^{2}, & & \text { in } \Omega \times(0, \infty), \\
& {\left[\left(\alpha_{1}+\alpha_{11} u+\alpha_{12} v\right) u\right]_{\gamma}+\beta_{1} u \Phi_{\gamma}=0,} & & \text { on } \partial \Omega \times(0, \infty), \\
& {\left[\left(\alpha_{2}+\alpha_{21} u+\alpha_{22} v\right) v\right]_{\gamma}+\beta_{2} v \Phi_{\gamma}=0,} & & \text { on } \partial \Omega \times(0, \infty),
\end{array}\right.
$$

where $\alpha_{i}(i=1,2)$ are positive constants, $\alpha_{i j}, \gamma_{i j}$ and $\beta_{i}, c_{i}(i, j=1,2)$ are nonnegative constants. $\Phi$ is the $C^{1}$-function; In the case, global existence results are shown when one of the following conditions holds:

\footnotetext{
*Received May 26, 1999; accepted for publication October 25, 2002.

${ }^{\dagger}$ Department of Mathematics, Armored Force Engineering Institute, Beijing 100072, P.R.of China (ywl-math@21cn.com).
} 
(1) $0<\alpha_{21}<8 \alpha_{11}$ and $0<\alpha_{12}<8 \alpha_{22}$;

(2) $\alpha_{11}>0, \alpha_{21}=\alpha_{22}=0$.

We should note that the situation is quite different from our problem where $\alpha_{11}=\alpha_{21}=0$.

In [4], Redinger given the global existence of the solution for the system (1.1) under the following assumption conditions

$$
\left\{\begin{array}{l}
(1) F(u, v) \equiv e_{1}-h(u)-d_{1} v, G(u, v) \equiv e_{2}-d_{2} u-b_{2} v ; \\
(2) h \in C^{2}(R, R), \text { and, lim inf } h(s) / s^{\nu}>0 . \\
\text { for some } \nu>1 \text {. } \\
\text { where the } e_{i}, d_{i} \text { and } b_{2} \text { are positive constants }
\end{array}\right.
$$

In this present paper, our main result can be stated as following

TheOrem. Assume $u_{0}$ and $v_{0}$ satisfy $\left(H_{0}\right)$, and, $F(u, v), G(u, v)$ to have the following properties:

$$
\left\{\begin{array}{l}
(1) F(u, v) \equiv e_{1}-h(u)-d_{1} v, G(u, v) \equiv e_{2}-d_{2} u-b_{2} v \\
(2) h \in C^{2}(R, R), \text { and, } h(s) \geq A_{0} s \\
\text { where the } e_{i}, d_{i}, A_{0} \text { and } b_{2} \text { are positive constants }
\end{array}\right.
$$

Then, systems (1.1) has a unique global solution.

To prove this result, we will work in the framework of $L_{p}$ and $W_{p}^{2,1}$ and employ the $L_{p}$-requarity theory for the linear parabolic equations. Our basic tools, which help us to derive some a priori estimates, are the duality technique and the Gronwall's inequality.

\section{Notation: (see [5][8])}

$Q_{T}=\Omega \times[0, T]$.

$L_{p}(\Omega)$ is the Banach space consisting of all measurable functions on $\Omega$ that are $p$ th-power $(p \geq 1)$ summable on $\Omega$. The norm in it is defined by the equations

$$
\|u\|_{p, \Omega}=\left(\int_{\Omega}|u(x)|^{p} d x\right)^{\frac{1}{p}} \text { and }\|u\|_{\infty, \Omega}=\operatorname{vrimax}|u| .
$$

$W_{p}^{2,1}\left(Q_{T}\right)(p \geq 1)$ is the Banach space consisting of the elements of $L_{p}\left(Q_{T}\right)$ having generalized derivatives of the form $D_{t}^{r} D_{x}^{s}$ with $r$ and $s$ satisfying the inequality $2 r+s \leq$ 2. The norm in it is defined by the equality

$$
\|u\|_{p, Q_{T}}^{(2)}=\sum_{2 r+s \leq 2}\left\|D_{t}^{r} D_{x}^{s} u\right\|_{p, Q_{T}} .
$$




\section{Preliminaries.}

2.1. Existence and uniqueness of local solutions. A local existence result for (1.1) is given by the following theorem (see Amann [6], Yagi [7]).

Lemma 2.1. Suppose that $u_{0}$ and $v_{0}$ satisfy $\left(H_{0}\right)$, then, there exists a positive constant $T^{*}$ such that (1.1) has a unique nonnegative solution $(u, v)$, s.t.: $\forall 0<T<$ $T^{*}$,

$$
u, v \in C\left((0, T] ; H^{2}(\Omega)\right) \cap C^{1}\left((0, T] ; L^{2}(\Omega)\right) .
$$

2.2. The (backward) adjoint equation. In order to use a duality technique to obtain estimates on $(u, v)$ in $W_{p}^{2,1}\left(Q_{T}\right)(p \geq 1)$, we need estimates on the solution $\chi$ of the (backward) adjoint equation

$$
\left\{\begin{array}{l}
\chi_{t}+a_{0} \triangle \chi+\theta=0, \text { in } Q_{T}, \\
\chi_{\gamma}=0, \text { on } \partial \Omega \times(0, T), \\
\chi(\cdot, T)=0, \text { in } \Omega
\end{array}\right.
$$

where $\theta \in L_{p}\left(Q_{T}\right)\left(q=\frac{p}{p-1}\right), \theta \geq 0 ; a_{0} \in C^{0, \lambda}\left(Q_{T}\right)$ for some $\lambda>0$,and, there exists a constant $\mu_{0}$,s.t.:

$$
a_{0}(x, t) \geq \mu_{0}, \text { for all }(x, t) \in Q_{T}
$$

We now state some well-known $L_{p}$-regularity results for (2.1).

Lemma 2.2. The adjoint equation (2.1) has a unique solution $\chi \in W_{p}^{2,1}\left(Q_{T}\right)$ with $\chi \geq 0$. If $\|\theta\|_{q, Q_{T}}=1$, then there exists a constant $C=C(p, T)$ independent of $\theta$, and, is continue to $T$, such that $\|\chi\|_{q, Q_{T}}^{(2)} \leq C$. Furthermore, $C$ can be chosen so that

$\left\{\begin{array}{l}\text { (i) If } p>2 \text {, then }\|\chi\|_{4 q /(4-q), Q_{T}} \leq C \text {, and, }\|\nabla \chi\|_{4 q /(4-q), Q_{T}} \leq C, \\ \text { (ii) }\|\chi(\cdot, 0)\|_{q, \Omega} \leq C \text {. }\end{array}\right.$

Proof. From [4](Theorem IV.9.1), there is a constant $C_{0}(p)$ independent $T$, such that

$$
\|\chi\|_{q, Q_{T}}^{(2)} \leq C_{0}(p)\|\theta\|_{q, Q_{T}}=C_{0}(p) .
$$

Furthermore, there is a constant $C_{1}(p)$ independent $T$. such that

$$
\|\operatorname{div} \chi\|_{q^{*}, Q_{T}} \leq C_{1}(p)\|\chi\|_{q, Q_{T}}^{(2)}
$$

where $q^{*}=4 q /(4-q)$ (see [4] lemma II 3.3).

Therefore, it follows that (2.3)(i) holds.

The same is true for (2.3)(ii) due again to [4] (lemma II 3.4). 
3. The estimates for $u$ and $v$. We note the nonnegativity of $u, v$ follows from theorem 2.1, and ,denote by $T^{*}$ a maximal existence time of the solution $(u, v)$ to (1.1)

LEMMA 3.1. If $T^{*}<\infty$, then, under the assumptions $\left(H_{0}\right)$ and (1.4), we have the following estimates

$$
\|v\|_{\infty, Q_{T^{*}}} \leq \text { Const. } \quad\|u\|_{4, Q_{T^{*}}} \leq \text { Const. }
$$

Proof. From the system (1.1), we see that $v$ satisfies

$$
\begin{cases}v_{t} \leq \triangle\left(a_{2} v\right)+e_{2} v-b_{2} v^{2}, & (x, t) \in \Omega \times\left(0, T^{*}\right) . \\ \delta v+(1-\delta) v_{\gamma}=0, & (x, t) \in \partial \Omega \times\left(0, T^{*}\right) . \\ v(x, 0)=v_{0}(x), & x \in \Omega .\end{cases}
$$

Hence, it follows from the comparison principle that $v$ is bounded from above by a constant $M_{0}$.i.e.

$$
\|v\|_{\infty, Q_{T^{*}}} \leq M_{0} \equiv \text { const. }
$$

Integrating (1.1)- $u$ equation over $Q_{T}\left(0<T<T^{*}\right)$, results in

$$
\begin{aligned}
\int_{\Omega} u(T) d x \leq & \int_{\Omega} u_{0}(x) d x+\int_{0}^{T} \int_{\partial \Omega}\left(a_{1} u+d u v\right)_{\gamma} \\
& +\int_{Q_{T}}\left[e_{1} u-h(u) u\right] d x d t \\
\leq & \int_{\Omega} u_{0}(x) d x+\int_{0}^{T} \int_{\partial \Omega}\left[\left(a_{1}+d v\right) u_{\gamma}+d u v_{\gamma}\right] \\
& +\int_{Q_{T}}\left(e_{1} u-A_{0} u^{2}\right) d x d t
\end{aligned}
$$

From the boundary condition of the system (1.1), we have that

$$
\int_{0}^{T} \int_{\partial \Omega}\left[\left(a_{1}+d v\right) u_{\gamma}+d u v_{\gamma}\right] \leq 0
$$

Thus, (3.2) becomes that

$$
\begin{aligned}
\int_{\Omega} u(T) d x+\int_{Q_{T}} A_{0} u^{2} d x d t & \leq \int_{\Omega} u_{0}(x) d x+\int_{Q_{T}} e_{1} u d x d t \\
& \leq \int_{\Omega} u_{0}(x) d x+\int_{Q_{T}} e_{1} u^{2} d x d t
\end{aligned}
$$

By use of Gronwall's inequality, (3.4) implies that

$$
\|u\|_{2, Q_{T^{*}}} \leq C\left(u_{0}, T^{*}\right) .
$$

Thus,

$$
g_{1}(x, t)=v\left(e_{2}-d_{2} u-b_{2} v\right) \in L_{2}\left(Q_{T^{*}}\right)
$$


and, $v$ solves the following equation

$$
\begin{cases}v_{t}=\triangle\left(a_{2} v\right)+g_{1}(x, t), & (x, t) \in \Omega \times\left(0, T^{*}\right) ; \\ \delta v+(1-\delta) v_{\gamma}=0, & (x, t) \in \partial \Omega \times\left(0, T^{*}\right) ; \\ v(x, 0)=v_{0}(x), & x \in \Omega .\end{cases}
$$

From [4], we have that

$$
v \in W_{2}^{2,1}\left(Q_{T^{*}}\right), \nabla v \in L_{4}\left(Q_{T^{*}}\right),
$$

From system (1.1), (3.1),(3.3) and (3.7),we known that $u$ solves the following equation

$$
\left\{\begin{array}{rlrl}
u_{t}=h_{1}(x, t) \triangle u+h_{2}(x, t) \nabla u & & \\
& +h_{3}(x, t) u+h_{4}(x, t), & & (x, t) \in \Omega \times\left(0, T^{*}\right) \\
\delta v+(1-\delta) v_{\gamma}=0, & & (x, t) \in \partial \Omega \times\left(0, T^{*}\right) \\
& u(x, 0)=u_{0}(x), & & x \in \Omega .
\end{array}\right.
$$

where,

$$
\left\{\begin{array}{l}
h_{1}(x, t) \equiv\left(a_{1}+d v\right) \in L_{\infty}\left(Q_{T^{*}}\right) \\
h_{2}(x, t) \equiv 2 d(\nabla v) \in L_{4}\left(Q_{T^{*}}\right) \\
h_{3}(x, t) \equiv d \triangle v+e_{1} \in L_{2}\left(Q_{T^{*}}\right) \\
h_{4}(x, t) \equiv-h(u) u-d_{1} v u \in L_{1}\left(Q_{T^{*}}\right)
\end{array}\right.
$$

Set,

$$
h_{4}^{*}(x, t) \equiv 0 .
$$

then,

$$
h_{4}(x, t) \leq h_{4}^{*}(x, t), \quad \forall(x, t) \in Q_{T^{*}}
$$

Let $u^{*}$ solves the following equation

$$
\left\{\begin{array}{rlrl}
u_{t}^{*}=h_{1}(x, t) \triangle u^{*}+h_{2}(x, t) \nabla u^{*} & \\
\quad+h_{3}(x, t) u^{*}+h_{4}^{*}(x, t), & & \forall(x, t) \in \Omega \times\left(0, T^{*}\right) \\
\delta u^{*}+(1-\delta) u_{\gamma}^{*}=0, & & (x, t) \in \partial \Omega \times\left(0, T^{*}\right) \\
u^{*}(x, 0)=u_{0}(x), & & x \in \Omega .
\end{array}\right.
$$

By use of the comparison principle, we known that

$$
0 \leq u \leq u^{*}, \quad \forall(x, t) \in Q_{T^{*}}
$$

From [4] and (3.11),we have that, $\forall \epsilon \in(0,1)$

$$
u^{*} \in W_{2-\epsilon}^{2,1}\left(Q_{T^{*}}\right) \subset W_{2-\epsilon}^{1}\left(Q_{T^{*}}\right) \subset L_{3(2-\epsilon) /(1+\epsilon)}\left(Q_{T^{*}}\right),
$$


Thus, taking $\epsilon=\frac{2}{7}$, we have that

$$
u^{*} \in L_{4}\left(Q_{T^{*}}\right) \Rightarrow u \in L_{4}\left(Q_{T^{*}}\right)
$$

Furthermore, we can also obtain the following estimate for $v$.

LEMmA 3.2. If $T^{*}<\infty$, then, under the assumptions $\left(H_{0}\right)$ and (1.4), there exists a positive constant $\lambda\left(T^{*}\right)>0$, s.t.

$$
v \in C^{0, \lambda\left(T^{*}\right)}\left(\bar{Q}_{T^{*}}\right)
$$

Proof. From the Lemma 3.1, we have that

$$
g_{1}(x, t) \equiv e_{2} v-d_{2} u v-b_{2} v^{2} \in L_{4}\left(Q_{T^{*}}\right) .
$$

Hence, $v$ also solves the following parabolic equation

$$
\begin{cases}v_{t}=a_{2} \Delta v+g_{1}(x, t), & \text { in } Q_{T^{*}} \\ \delta v+(1-\delta) v_{\gamma}=0, & \text { on } \partial \Omega \times\left(o, T^{*}\right) \\ v(x, 0)=v_{0}(x), & \text { in } \Omega\end{cases}
$$

From [4], we have that

$$
v \in W_{4}^{2,1}\left(Q_{T^{*}}\right) \subset W_{4}^{1}\left(Q_{T^{*}}\right)
$$

By use the embedding theorem (see [4]), there exists a positive constant $\lambda\left(T^{*}\right)>0$ (Notice that $4>N+1=3$ ), s.t.

$$
W_{4}^{1}\left(Q_{T^{*}}\right) \subset C^{0, \lambda\left(T^{*}\right)}\left(\overline{Q_{T^{*}}}\right)
$$

We have completed the proof of lemma 3.2

We are now ready to derive an important estimate for $u$. Our main methods are the duality technique and the Hölder inequality.

We first give two lemmas.

LEMmA 3.3. Under the hypotheses $\left(H_{0}\right)$ and (1.4), if $T^{*}<\infty$, then, for all $p>1$, we have

$$
\|u\|_{p, Q_{T^{*}}} \leq C\left(p, T^{*}\right)
$$

Proof. Let $\varphi$ satisfy the following backward equation

$$
\left\{\begin{array}{l}
\varphi_{t}+\left(a_{1}+d v\right) \triangle \varphi+\theta=0 \text { in } Q_{T} \\
\varphi_{\gamma}=0 \text { on } \partial \Omega \times(0, T) \\
\varphi(\cdot, T)=0 \text { in } \Omega
\end{array}\right.
$$


Where, $\theta \in L_{q}\left(Q_{T}\right)\left(q=\frac{p}{p+1}, p>2\right)$ with $\theta \geq 0$ and $\|\theta\|_{q, Q_{T}}=1,(u, v)$ is the local solution of (1.1) in $\Omega \times\left[0, T^{*}\right), 0<T<T^{*}$.

Multiplying $u$ - equation of (1.1) by $\varphi$ and integrating the product in $Q_{T}$, we have

$$
\int_{Q_{T}} \varphi u_{t} d x d t=\int_{Q_{T}} \varphi \triangle\left[\left(a_{1}+d v\right) u\right] d x d t+\int_{Q_{T}} \varphi u\left[e_{1}-h(u)-d_{1} v\right] d x d t
$$

i.e.

$$
\begin{aligned}
& -\int_{Q_{T}} u \varphi_{t} d x d t-\int_{\Omega} \varphi(\cdot, 0) u_{0} d x \\
\leq & \int_{\partial \Omega \times(0, T)}\left\{\varphi\left[\left(a_{1}+d v\right) u\right]_{\gamma}-\left(a_{1}+d v\right) u \varphi_{\gamma}\right\} \\
& +\int_{Q_{T}}\left(a_{1}+d v\right) u \triangle \varphi d x d t+\int_{Q_{T}} e_{1} \varphi u d x d t,
\end{aligned}
$$

From the lemma 3.2, and the boundary condition of the system (1.1),and (3.15), we obtain that

$$
\int_{Q_{T}} \theta u d x d t \leq \int_{\Omega} \varphi(\cdot, 0) u_{0} d x+\int_{Q_{T}} e_{1} \varphi u d x d t
$$

By lemma 3.1, (3.18) becomes that

$$
\int_{Q_{T}} \theta u d x d t \leq C\left(u_{0}, p, T\right)+C\left(M_{0}, p, T\right)\|u\|_{4 p /(4+p), Q_{T}} .
$$

We write

$$
H(\lambda)=\frac{1}{1-\lambda}\left[\frac{4 p}{4+p}-\lambda p\right], \lambda \in\left(0, \frac{4}{4+p}\right),
$$

then

$$
\frac{d H(\lambda)}{d \lambda}=\frac{1}{(1-\lambda)^{2}} \cdot \frac{-p^{2}}{4+p}<0
$$

and

$$
H\left(\frac{4}{4+p}\right)=0
$$

Therefore, there exists $\lambda_{0}$, s.t.

$$
H\left(\lambda_{0}\right) \leq 1, \lambda_{0} \in\left(0, \frac{4}{4+p}\right) .
$$




$$
\begin{aligned}
\|u\|_{4 p /(4+p), Q_{T}} & =\left\|u^{\frac{4 p}{4+p}-\lambda_{0} p+\lambda_{0} p}\right\|_{1, Q_{T}}^{(4+p) / 4 p} \\
& =\left\|u^{\left(1-\lambda_{0}\right) H\left(\lambda_{0}\right)} u^{\lambda_{0} p}\right\|_{1, Q_{T}}^{(4+p) / 4 p} \\
& =\left\|u^{H\left(\lambda_{0}\right)}\right\|_{1, Q_{T}}^{\left(1-\lambda_{0}\right)(4+p) / 4 p}\left\|u^{p}\right\|_{1, Q_{T}}^{\lambda_{0}(4+p) / 4 p},
\end{aligned}
$$

while by lemma 3.1 and (3.23) it is seen that

$$
\left\|u^{H\left(\lambda_{0}\right)}\right\|_{1, Q_{T}}^{\left(1-\lambda_{0}\right)(4+p) / 4 p} \leq C_{p}\left(T^{*}\right), 0<T<T^{*},
$$

where $C_{p}\left(T^{*}\right)$ is a positive constant depending on $T^{*}$ and $p$.

Hence, (3.23) becomes

$$
\|u\|_{4 p /(4+p), Q_{T}} \leq C_{p}\left(T^{*}\right)\left\|u^{p}\right\|_{1, Q_{T}}^{\lambda_{0}(4+p) / 4 p},
$$

this then shows, in view of (3.25), that we can conclude from (3.19) that

$$
\begin{aligned}
\int_{Q_{T}} \theta u d x d t & \leq C\left(u_{0}, p, T\right)+C\left(M_{0}, p, T\right)\|u\|_{4 p /(4+p), Q_{T}} \\
& \leq C\left(u_{0}, p, T\right)+C\left(M_{0}, p, T^{*}\right)\|u\|_{p, Q_{T}}^{\lambda_{0}(4+p) / 4}
\end{aligned}
$$

From the process of the proof, it is easy to see that we can choose $C\left(u_{0}, p, T\right)$ and $C\left(M_{0}, p, T^{*}\right)$ are continuous functions depending on $T$. Therefore, (3.26) can be written that

$$
\int_{Q_{T}} \theta u d x d t \leq C\left(u_{0}, p, T^{*}\right)+C\left(M_{0}, p, T^{*}\right)\|u\|_{p, Q_{T}}^{\lambda_{0}(4+p) / 4} .
$$
that

Noting that $\lambda_{0} \in\left(0, \frac{4}{4+p}\right), \theta \in L_{q}\left(Q_{T}\right)$ is arbitrary and $\theta \geq 0$, then, (3.24) implies

$$
\|u\|_{p, Q_{T}} \leq C\left(u_{0}, p, T^{*}\right), \forall 0<T<T^{*} .
$$

Thus, we can obtain the following estimates for $u$

$$
\|u\|_{p, Q_{T^{*}}} \leq C\left(u_{0}, p, T^{*}\right) .
$$

We complete the proof.

We now establish the a priori estimates in $W_{p}^{2,1}\left(Q_{T^{*}}\right)$.

LEMMA 3.4. Under the hypotheses of lemma 3.3, we have

$$
u, v \in W_{p}^{2,1}\left(Q_{T^{*}}\right), \forall p>1 \text {. }
$$

Proof. Because of lemma 3.3, we have that $g_{1}(x, t) \in L_{p}\left(Q_{T^{*}}\right)(p>1)$, and , $v$ solves the equation (3.14). From [5], we have that

$$
v \in W_{p}^{2,1}\left(Q_{T^{*}}\right), \forall p>1 .
$$

From $u$-equation of systems (1.1), we known that $u$ solves equation (3.10). By use of the lemma 3.3, we have also that

$$
u \in W_{p}^{2,1}\left(Q_{T^{*}}\right), \quad \forall p>1
$$


4. The existence of global solutions. Now, we can begin to prove the existence theorem of global solutions.

TheOREM 4.1. Assume that $\left(H_{0}\right)$ and (1.4) are satisfied, then, (1.1) has a unique solution in $\Omega \times(0, \infty)$.

Proof. The proof consists of four steps.

\section{Step 1:}

Suppose for contradiction that $T^{*} i \infty$, from lemma 3.4, it follows that

$$
u, v \in W_{p}^{2,1}\left(Q_{T^{*}}\right), \forall p>1 \text {. }
$$

Now, let us differentiate the $u$-equation of (1.1) in $t$, and take the product with $\left(a_{1}+d v\right) u_{t}$. Integrating the product on $\Omega \times\left[\delta_{0}, t\right]$ with a fixed $\delta_{0}\left(0<\delta_{0}<t<T^{*}\right)$, we obtain that

$$
\begin{aligned}
& \frac{1}{2} \int_{\Omega \times\left[\delta_{0}, t\right]}\left(a_{1}+d v\right)\left(u_{t}^{2}\right)_{t} \\
= & \int_{\Omega \times\left[\delta_{0}, t\right]}\left(a_{1}+d v\right) u_{t}\left\{\Delta\left[\left(a_{1}+d v\right) u\right]\right\}_{t} \\
& +\int_{\Omega \times\left[\delta_{0}, t\right]}\left(a_{1}+d v\right) u_{t}\left(e_{1} u_{t}-u h^{\prime}(u) u_{t}-d_{1} v u_{t}-d u v_{t}\right) .
\end{aligned}
$$

From (4.1), the last term of (4.2) has the following estimate

$$
\int_{\Omega \times\left[\delta_{0}, t\right]}\left(a_{1}+d v\right) u_{t}\left(e_{1} u_{t}-u h^{\prime}(u) u_{t}-d_{1} v u_{t}-d u v_{t}\right) \leq C\left(p, T^{*}, \delta_{0}\right) .
$$

We have that the (4.2) becomes that

$$
\begin{aligned}
& \frac{1}{2} \int_{\Omega \times\left[\delta_{0}, t\right]}\left(a_{1}+d v\right)\left(u_{t}^{2}\right)_{t} \\
\leq & C\left(p, T^{*}, \delta_{0}\right)+\int_{\partial \Omega \times\left[\delta_{0}, t\right]}\left(a_{1}+d v\right) u_{t}\left[\left(a_{1}+d v\right) u_{\gamma}+d u v_{\gamma}\right]_{t} \\
- & \int_{\Omega \times\left[\delta_{0}, t\right]} \nabla\left[\left(a_{1}+d v\right) u_{t}\right] \nabla\left[\left(a_{1}+d v\right) u_{t}\right. \\
= & C\left(p, T^{*}, \delta_{0}\right)+\int_{\partial \Omega \times\left[\delta_{0}, t\right]}\left(a_{1}+d v\right) u_{t}\left\{\left(a_{1}+d v\right)\left(u_{\gamma}\right)_{t}+d v_{t} u_{\gamma}+d u_{t} v_{\gamma}+d u\left(v_{\gamma}\right)_{t}\right\} \\
- & \int_{\Omega \times\left[\delta_{0}, t\right]}\left[\left(a_{1}+d v\right) \nabla u_{t}+d u_{t} \nabla v\right]\left[\left(a_{1}+d v\right) \nabla u_{t}+d v_{t} \nabla u+d u_{t} \nabla v+d u \nabla v_{t}\right] .
\end{aligned}
$$

Next, let us differentiate the second equation in (1.1) in $t$, and, multiplying by $\left(1+u^{2}\right) v_{t}$, and integrating the product in $\Omega \times\left[\delta_{0}, t\right]$, we can obtain that

$$
\begin{aligned}
& \frac{1}{2} \int_{\Omega}\left(1+u^{2}\right) v_{t}^{2}(t) \\
\leq & \int_{\Omega \times\left[\delta_{0}, t\right]} a_{2}\left(1+u^{2}\right) v_{t} \Delta v_{t}+\int_{\Omega \times\left[\delta_{0}, t\right]}\left(1+u^{2}\right) v_{t}\left(e_{2} v_{t}-d_{2} u v_{t}-d_{2} v u_{t}-2 b_{2} v v_{t}\right) \\
\leq & C\left(p, T^{*}, \delta_{0}\right)+\int_{\partial \Omega \times\left[\delta_{0}, t\right]} a_{2}\left(1+u^{2}\right) v_{t}\left(v_{\gamma}\right)_{t}-\int_{\Omega \times\left[\delta_{0}, t\right]} a_{2} \nabla\left[\left(1+u^{2}\right) v_{t}\right] \nabla v_{t} .
\end{aligned}
$$


From the boundary conditions of systems (1.1), we have that

$$
\left\{\begin{array}{l}
(1) \delta=1: u=v=0 \Rightarrow u_{t}=v_{t}=0, \forall(x, t) \in \partial \Omega \times\left[\delta_{0}, t\right] \\
(2) \delta=0: u_{\gamma}=v_{\gamma}=0 \Rightarrow\left(u_{\gamma}\right)_{t}=\left(v_{\gamma}\right)_{t}=0, \forall(x, t) \in \partial \Omega \times\left[\delta_{0}, t\right]
\end{array}\right.
$$

Then, (4.4) (4.5) become that

$$
\begin{aligned}
& \frac{1}{2} \int_{\Omega \times\left[\delta_{0}, t\right]}\left(a_{1}+d v\right)\left(u_{t}^{2}\right)_{t} \leq C\left(p, T^{*}, \delta_{0}\right)- \\
& -\int_{\Omega \times\left[\delta_{0}, t\right]}\left[\left(a_{1}+d v\right) \nabla u_{t}+d u_{t} \nabla v\right]\left[\left(a_{1}+d v\right) \nabla u_{t}+d v_{t} \nabla u+d u_{t} \nabla v+d u \nabla v_{t}\right] \\
& \frac{1}{2} \int_{\Omega}\left(1+u^{2}\right) v_{t}^{2}(t) \leq C\left(p, T^{*}, \delta_{0}\right)-\int_{\Omega \times\left[\delta_{0}, t\right]} a_{2} \nabla\left[\left(1+u^{2}\right) v_{t}\right] \nabla v_{t}
\end{aligned}
$$

we known that

$$
\begin{aligned}
& -\int_{\Omega \times\left[\delta_{0}, t\right]}\left[\left(a_{1}+d v\right) \nabla u_{t}+d u_{t} \nabla v\right]\left[\left(a_{1}+d v\right) \nabla u_{t}+d v_{t} \nabla u+d u_{t} \nabla v+d u \nabla v_{t}\right] \\
= & -\int_{\Omega \times\left[\delta_{0}, t\right]}\left\{\left(a_{1}+d v\right)^{2}\left|\nabla u_{t}\right|^{2}+\left(a_{1}+d v\right)\left[d v_{t} \nabla u+2 d u_{t} \nabla v\right.\right. \\
& \left.+d\left(a_{1}+d v\right) u \nabla v_{t} \nabla u_{t}+d^{2} u_{t} \nabla v u \nabla v_{t}\right\} \\
\leq & -\int_{\Omega \times\left[\delta_{0}, t\right]}(1-\varepsilon)\left(a_{1}+d v\right)^{2}\left|\nabla u_{t}\right|^{2}- \\
& -d\left(a_{1}+d v\right) u \nabla u_{t} \nabla v_{t}-\varepsilon \int_{\Omega \times\left[\delta_{0}, t\right]}\left|\nabla\left(v_{t}\right)\right|^{2}+C\left(p, T^{*}, \delta_{0}, \varepsilon\right)
\end{aligned}
$$

here, $\varepsilon \in(0,1)$ is arbitrary.

For (4.7), we have the similar estimates

$$
\begin{aligned}
& -\int_{\Omega \times\left[\delta_{0}, t\right]} a_{2} \nabla\left[\left(1+u^{2}\right) v_{t}\right] \nabla v_{t} \\
\leq & -a_{2}(1-\varepsilon) \int_{\Omega \times\left[\delta_{0}, t\right]}\left[\left(1+u^{2}\right)\left|\nabla v_{t}\right|^{2}+C\left(p, T^{*}, \delta_{0}, \varepsilon\right) .\right.
\end{aligned}
$$

From, (4.7)-(4.11), we obtain that, for any $\delta_{0}<t<T^{*}, \varepsilon \in(0,1), k>0$

$$
\begin{aligned}
& \frac{1}{2} \int_{\Omega}\left(a_{1}+d v\right) u_{t}^{2}(t)+\frac{k}{2} \int_{\Omega}\left(1+u^{2}\right) v_{t}^{2}(t) \\
\leq & -\int_{\Omega \times\left[\delta_{0}, t\right]}\left\{(1-\varepsilon)\left(a_{1}+d v\right)^{2}\left|\nabla u_{t}\right|^{2}+d\left(a_{1}+d v\right) u \nabla u_{t} \nabla v_{t}+\varepsilon \int_{\Omega \times\left[\delta_{0}, t\right]}\left|\nabla\left(v_{t}\right)\right|^{2}\right. \\
& -k a_{2}(1-\varepsilon) \int_{\Omega \times\left[\delta_{0}, t\right]}\left[\left(1+u^{2}\right)\left|\nabla v_{t}\right|^{2}+C\left(p, T^{*}, \delta_{0}\right) .\right. \\
= & -\int_{\Omega \times\left[\delta_{0}, t\right]}\left\{(1-\varepsilon)\left(a_{1}+d v\right)^{2}\left|\nabla u_{t}\right|^{2}+d\left(a_{1}+d v\right) u \nabla u_{t} \nabla v_{t}\right. \\
& \left.+\left[k a_{2}(1-\varepsilon)-\varepsilon\right]\left(1+u^{2}\right)\left|\nabla v_{t}\right|^{2}\right\}+C\left(p, T^{*}, \delta_{0}\right) .
\end{aligned}
$$


Taking $\varepsilon=\min \left\{\frac{1}{2}, \frac{d^{2}}{2}\right\}, k=\frac{4 d^{2}}{a_{2}}$, we have that

$$
\begin{aligned}
& \frac{1}{2} \int_{\Omega}\left(a_{1}+d v\right) u_{t}^{2}(t)+\frac{d^{2}}{a_{2}} \int_{\Omega}\left(1+u^{2}\right) v_{t}^{2}(t) \leq-\int_{\Omega \times\left[\delta_{0}, t\right]} \frac{1}{2}\left(a_{1}+d v\right)^{2}\left|\nabla u_{t}\right|^{2} \\
& -\int_{\Omega \times\left[\delta_{0}, t\right]} d\left(a_{1}+d v\right) u \nabla u_{t} \nabla v_{t}+d^{2}\left(1+u^{2}\right)\left|\nabla v_{t}\right|^{2}+C\left(p, T^{*}, \delta_{0}\right)
\end{aligned}
$$

i.e.

$$
\begin{aligned}
& \frac{1}{2} \int_{\Omega}\left(a_{1}+d v\right) u_{t}^{2}(t)+\frac{d^{2}}{a_{2}} \int_{\Omega}\left(1+u^{2}\right) v_{t}^{2}(t) \\
& +\int_{\Omega \times\left[\delta_{0}, t\right]}\left\{\frac{1}{16}\left(a_{1}+d v\right)^{2}\left|\nabla u_{t}\right|^{2}+\int_{\Omega \times\left[\delta_{0}, t\right]} \frac{d^{2}}{8}\left(1+u^{2}\right)\left|\nabla v_{t}\right|^{2} \leq C\left(p, T^{*}, \delta_{0}\right) .\right.
\end{aligned}
$$

\section{Step 2:}

Multiplying the first equation of (1.1) by $u^{p}$ and integrating the product in $Q_{t}(0<$ $\left.t<T^{*}\right)$, we have

$$
\begin{aligned}
\frac{1}{p+1} \int_{\Omega} u^{p+1}(t)= & \frac{1}{p+1} \int_{\Omega} u_{0}^{p+1}+\int_{Q_{t}} u^{p} \Delta\left[\left(a_{1}+d v\right) u\right] \\
& +\int_{Q_{t}} u^{p+1}\left\{e_{1}-h(u)-d_{1} v\right\}
\end{aligned}
$$

$($ from lemma 3.4$) \leq C\left(u_{0}, v_{0}, p, T^{*}\right)$

From the second equation of (1.1), a similar inequality for $v$ is obtained

$$
\frac{1}{p+1} \int_{\Omega} v^{p+1}(t) \leq C\left(u_{0}, v_{0}, p, T^{*}\right) .
$$

Hence, from the systems (1.1) and the above estimates, we have, for all $0<t<T^{*}$,

$$
\left\|\left\{\Delta\left[\left(a_{2}+d v\right) u\right]\right\}(t)\right\|_{2, \Omega}+\|(\Delta v)(t)\|_{2, \Omega} \leq C\left(u_{0}, T^{*}\right) .
$$

Furthermore, differentiating the $v$-equation in (1.1) in $t$, and take the scalar product with $v_{t}^{2 p+1}(p \geq 1)$. Then integration of the product on $[0, t]\left(0<t<T^{*}\right)$ yields that

$$
\left\|\left(v_{t}\right)(t)\right\|_{2 p+2, \Omega} \leq C\left(u_{0}, p, T^{*}\right), \text { for all } 0<t<T^{*},
$$

thus

$$
\|(\triangle v)(t)\|_{2 p+2, \Omega} \leq C\left(u_{0}, p, T^{*}\right), \text { for all } 0<t<T^{*} .
$$

In view of the embedding theorem

$$
H^{1}(\Omega)=W_{2}^{1}(\Omega) \subset L_{4}(\Omega) .
$$


We have that

$$
\begin{cases}\left\|\left\{\nabla\left[\left(a_{2}+d v\right) u\right]\right\}(t)\right\|_{4, \Omega} \leq C\left(u_{0}, v_{0}, T^{*}\right), & \text { for all } 0<t<T^{*}, \\ \|(\nabla v)(t)\|_{p, \Omega} \leq C\left(u_{0}, v_{0}, T^{*}\right), & \text { for all } 0<t<T^{*}\end{cases}
$$

Thus

$$
\|(\nabla u)(t)\|_{p, \Omega} \leq C\left(u_{0}, v_{0}, T^{*}\right) \text {, for all } 0<t<T^{*},
$$

Noting that

then

$$
|\Delta u| \leq\left|\left(a_{2}+d v\right) \Delta u\right| \leq\left|\Delta\left[\left(a_{2}+d v\right) u\right]\right|+2 d|\nabla u \nabla v|+d|u \triangle v|
$$

$$
\begin{aligned}
& \|\Delta u\|_{2, \Omega}^{2} \leq \| \Delta\left[\left(a_{2}+d v\right) u \|_{2, \Omega}^{2}+4 d^{2}\left(\|\nabla u\|_{4, \Omega}^{4}+\|\nabla v\|_{4, \Omega}^{4}\right)\right. \\
& +d^{2}\left(\|u\|_{4, \Omega}^{4}+\|\Delta v\|_{4, \Omega}^{4}\right) \leq C\left(u_{0}, T^{*}\right), \text { for all } 0<t<T^{*},
\end{aligned}
$$

Hence, the norms $\|u(t)\|_{H^{2}(\Omega)}$ and $\|v(t)\|_{H^{2}(\Omega)}$ remain bounds as $t \rightarrow T^{*}$.

\section{Step 3:}

From [8], then the solution $u, v$, can be extended to a nonnegative solution beyond the $T^{*}$. Hence, (1.1) has a global solution, we complete the proof.

\section{REFERENCES}

[1] Shigesada N., Kawasaki K., \& Teramoto E., Spatial segregation of interacting species, J.Theor. Biol., 79 (1979), pp. 83-99.

[2] Mimura M., Stationary pattern of some density-dependent diffusion system with competitive dynamics, Hiroshima Math. J., 11 (1981), pp. 621-635.

[3] YAGI A., Global solution to some quasilinear parabolic systems in population dynamics, Nonlinear Analysis, 21 (1993), pp. 603-630.

[4] Redinger R., Existence of the Global Attractor for a Strongly Coupled Parabolic SystemArising in Population Dynamics, J. of D.E., 118 (1995), pp. 219-252.

[5] Ladyzenskaja O.A., Solonnikov V.A.\& Uralceva N.N., Linear and quasilinear equations of parabolic type, Am. Math. Soc., Providence, R.I., (1968).

[6] Amann H., Quasilinear evolution equations and parabolic systems, Trans. Am. Math. Soc., 293 (1986), pp. 191-227.

[7] Yagi A., Abstract quasilinear evolution equations of parabolic type in Banach space, Bollettino U.M.I., (7)5-B (1991), pp. 341-368.

[8] Adams R.A., Sobolev spaces, Academic press, New York, (1975).

[9] YANG W.L., On the question of global existence of reaction-diffusion systems withmixed boundary conditions, Ph.D. Thesis, Lanzhou university, (1995). 